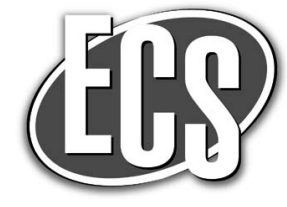

\title{
Laser Diode Induced Lighting Modules
}

\author{
A. Salimian, ${ }^{\text {Z G. R. Fern, }{ }^{*} \text { H. Upadhyaya, and J. Silver* }}$
}

Centre for Phosphors and Display Materials, Wolfson Centre for Material Processing, Institute for Materials and Manufacturing, Brunel University London, Uxbridge, Middlesex UB8 3PH, United Kingdom

\begin{abstract}
Laser diodes have the potential of becoming the light engines of future lighting technology since they have negligible efficiency droop factor, unlike light emitting diodes. This study demonstrates the possibility of laser diodes coupled to phosphor targets being used as a solid state lighting system with high power applications. It was revealed that white light emitting modules with efficiency of up to 217 lumens per watt based on laser diodes can currently be made and upon further development of laser diode technology and relevant phosphor materials there is room for further improvements. The report also demonstrates the ability of this technology to produce a tailored emission spectrum for a given specific requirement. Two test lamp prototypes were made using laser diodes and phosphor targets and their emission characteristics were investigated.

(C) The Author(s) 2016. Published by ECS. This is an open access article distributed under the terms of the Creative Commons Attribution 4.0 License (CC BY, http://creativecommons.org/licenses/by/4.0/), which permits unrestricted reuse of the work in any medium, provided the original work is properly cited. [DOI: 10.1149/2.0101603jss] All rights reserved.
\end{abstract}

Manuscript submitted October 22, 2015; revised manuscript received December 17, 2015. Published January 5, 2016.

Currently light emitting diodes (LEDs) coupled with phosphors are the most efficient source of solid state white light provided that they are operated at low input powers. The drop in the LED's efficiency at higher input powers is associated to a phenomenon called the efficiency droop. This phenomenon has been investigated for the past decade and is thought to be linked to the Auger effect. ${ }^{1-3}$ Despite extensive research in to the field no fundamental or definitive solution has been put forward to eliminate this effect. To achieve more lumens per power consumed, the LEDs are typically operated under higher input powers at the expense of efficiency.

An alternative approach is to use Laser Diodes (LDs) in combination with the same phosphors applied in LED lighting but packaged differently to achieve high performance and efficient solid state lighting (SSL). The LDs are operated under stimulated emission and the mechanisms of efficiency droop are clamped at their lasing threshold. ${ }^{4}$ This concludes that through application of LDs, higher efficiencies at higher input power densities can be achieved. The LDs are more efficient in this respect because they are operated by stimulated emission. At lasing threshold, all the recombination processes (The Auger, Shockley-Read-Hall mechanism \& spontaneous) are clamped and additional carriers injected into the light quantum wells contribute only to stimulated emission, so it can be argued that unless a fix to the efficiency droop which is an intrinsic issue is found, the LDs will be competing with LEDs for the future of high brightness/high power SSL modules. ${ }^{5}$ Figure 1 demonstrates a comparison of the power conversion efficiency (PCE) of LDs and LEDs and what is expected in terms of performance improvement from them in future. Future LEDs will require modules which have a shift in PCE to higher input power densities. Considering that Auger recombination is a fundamental process, improvements in LED structural design such as thicker active regions and no polar substrates will only be able to offset the efficiency droop but not eliminate it.

However the LDs have certain issues related to their application which need to be addressed prior to them becoming the light engines for future SSLs. Their existing peak PCE of $\sim 30 \%$ is well below the $69 \%$ PCE of LEDs. Simulation studies have been carried out to generate results as an indication of what the future LDs and LEDs can offer in terms of luminous efficacy of radiation. ${ }^{6}$ Apart from the required advancement in LD technology, it's important that the phosphor screens/targets which are to be stimulated by high power LDs can withstand the high temperature build up resulting from exposure to the laser beam and to be constructed in a way so that this thermal energy is managed. Further work is required to assess the current phosphor materials in terms of their application in LD based SSLs. The nature of the phosphor material, its morphology and crystal structure need to be investigated to obtain efficient targets for the LDs.

\footnotetext{
*Electrochemical Society Active Member.
}

${ }^{\text {zE} E-m a i l: ~ a l i . s a l i m i a n @ b r u n e l . a c . u k ~}$
We previously reported on our solution for the thermal management of phosphor targets which can be exposed to laser beam powers of up to $5 \mathrm{~W}^{7}$ In this report we looked into comparing two different commercial phosphors; both based on the yttrium aluminum garnet system but from two separate commercial suppliers. The suppliers do not declare the exact chemical compositions. The phosphors were used to create targets for our lamp so that the efficiency of the lamp can be evaluated based on the type of the phosphor material used.

\section{Experimental}

The phosphor materials used in this work were based on the yttrium aluminum garnet structure. The phosphor materials (A and B) were obtained from two separate commercial suppliers. The phosphors were then embedded in a matrix exhibiting high thermal conductivity and were constructed in to the target module of the laser lamp which we built and reported on previously. ${ }^{7}$ The composition and synthesis of the matrix-phosphor system developed by the authors cannot be disclosed at present; however in terms of thermal characteristics the system has a thermal conductivity of $26 \mathrm{~W} \mathrm{~m}^{-1} \mathrm{k}^{-1}$. Total thickness of the circular phosphor targets prepared using this system was $5 \mathrm{~mm}$ with a diameter of $15 \mathrm{~mm}$. The precise synthesis route and composition of the phosphors are not disclosed by the suppliers hence the phosphor particles were assessed using scanning electron microscopy (SEM) coupled with energy dispersive X-ray spectroscopy (EDS), X-ray powder diffraction (XRD) and the distribution of the phosphor particles within the matrix was assessed using laser Raman spectroscopy.

Considering the high cost of the phosphors, the lesser amount used would lead to a more economical device. Hence to identify the optimum phosphor quantity to use in the target, varying amounts of phosphor (percentage by mass) to matrix material was used to establish the most commercially/performance optimized quantity of phosphor to use. Also by mixing and combining different phosphors with peak emissions at 530 and $650 \mathrm{~nm}$ we demonstrate the capability of LD based SSLs as suitable substitutes in future light sources requiring custom emission signature or broad emission spectrum, notably as a light source for solar cell simulation applications and as a possible alternative to natural indoor lighting. Photometric analysis of the emission was carried out by placing the test lamps inside an integrating sphere; the sphere was a 'Labsphere LMS200' model with sphere diameter of 20 inch. This sphere is capable of analyzing test lamps with output of up to $400 \mathrm{~W}$ or 8000 lumens. The sphere was calibrated using an AS-01331-000 test lamp provided by the manufacturer. The correction to account for light absorbed by the test lamp body was carried out using an auxiliary lamp inside the sphere which calculated the light absorbed by the test lamp body which was automatically factored into the measurements. 

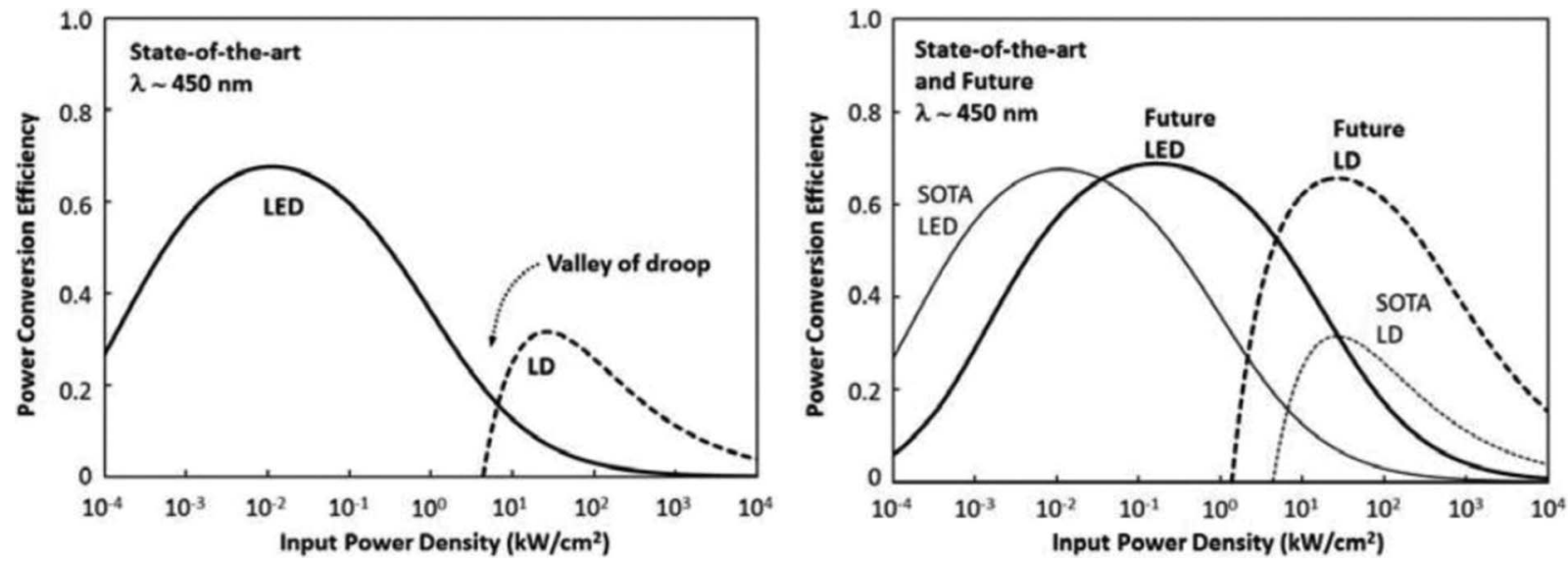

Figure 1. Left: Power conversion efficiency versus input power density of state of the art (SOTA) blue LED and LD. Right: Power conversion efficiency versus input power density of the SOTA and future blue LEDs and LDs. (Ref. 5 with permission from authors).

\section{Test Lamps}

Two test lamps were made using the type A and B phosphor containing $10 \%$ phosphor by mass. The laser diode type that was used to construct the test lamps was a Nichia NDB7A75 that delivered $3.5 \mathrm{~W}$ of laser beam when fitted with a standard 'three element lens' which reduces the beam output by up to $40 \%$, hence maximum of $3.5 \mathrm{~W}$ is achieved when the basic lens is used. To avoid this loss and utilize most of the beam energy generated by the diode our module was constructed by using a G2 lens (Figure 2) from 'JTECH PHOTONICS Inc.' The G2 allows achieving up to $>35 \%$ more beam energy however with certain restrictions in controlling the beam divergence over long distance $(>50 \mathrm{~cm})$. We could achieve up to $6 \mathrm{~W}$ of beam power by running the diodes at higher current thresholds, however on most of the tests we operated the diode so that it generated $5 \mathrm{~W}$ of beam energy.

Lamp Alpha.-This lamp was made similar to the previous test lamp we reported. The module was made using a single $5 \mathrm{~W}$ laser source, a poly acrylic tube and phosphor target with a small heat sink. This lamp was placed inside the integrating sphere and the emission was analyzed for a period of fourteen minutes at one minute intervals. The lamp's metal parts were reflective in color hence ensuring minimum error when placed inside the sphere. The objective of this lamp is to be able to characterize individual phosphor targets separately. By loading each of the A and B phosphors targets into this lamp some comparative data were obtained on the performance of each phosphor type and the maximum output of each was calculated. The design and structure of this lamp is presented in Figure 3.

Lamp Beta.-This lamp was constructed by machining a housing module where three $5 \mathrm{~W}$ laser diodes along with three phosphor targets made with Phosphor B (10\% content) were integrated. The module was then connected to a fan assisted heat sink. The objective of this design was to create a light engine that could be used to test our phosphor targets under stable temperatures over a long period of time. The size of the module was too large for our integrating sphere and the body color was dark, hence the read out from the integrating sphere had to be adjusted based on data obtained from the simpler type Alpha lamp. Basically each of the 'three' targets that were placed in this lamp were first evaluated using the lamp 'Alpha'. Each phosphor target on average demonstrated a maximum output of 800 lumens when tested using lamp 'Alpha'. When all three targets were activated in lamp 'Beta' configuration inside the sphere, the maximum read out was $1338 \mathrm{~lm}$, however as mentioned due to the size of the lamp 'Beta' and the location where targets and each according laser diode was housed (Figure 3B), significant amount of the emission was unaccounted for. The lamp 'Beta' as mentioned was designed with a cooling heat sink to enable us measuring the emission characteristic of the targets under stable temperatures over extended time, hence enabling us having a comparative measure to the data obtained from phosphor targets in lamp 'Alpha'. The objective of this study was characterising the performance of the 'phosphor targets' and not the lamps; hence the 1338 lumen reading from lamp 'Beta' (fitted with three targets) was corrected according to the following logic:

a. 1 phosphor target in Lamp 'Alpha': 800 Lumens.

Hence: $\sim 800$ lumens per target.

b. Same phosphor target in Lamp 'Beta', same Laser diode: $~ 435$ lumens.

Hence: 365 lumen is lost due to size of the lamp and the phosphor target housing.

c. Three phosphor targets in lamp 'Beta': 1338 Lumen

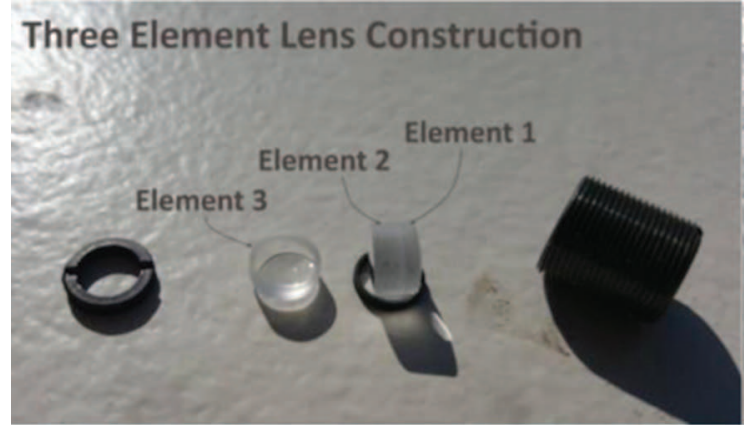

\section{High Efficiency Lens (G2) Constrüction}

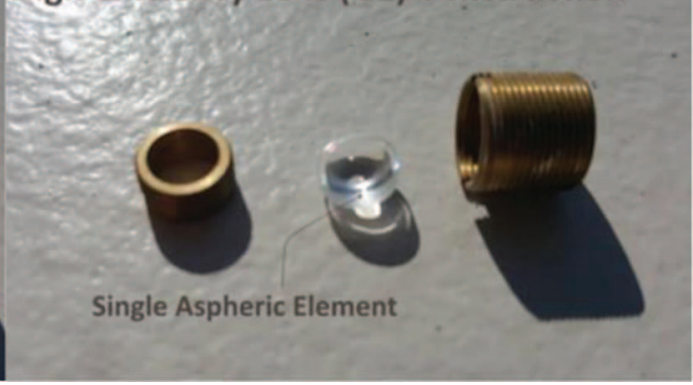

Figure 2. Comparing the structure of the 3 element lens and a G2 lens (JTECH photonics). 

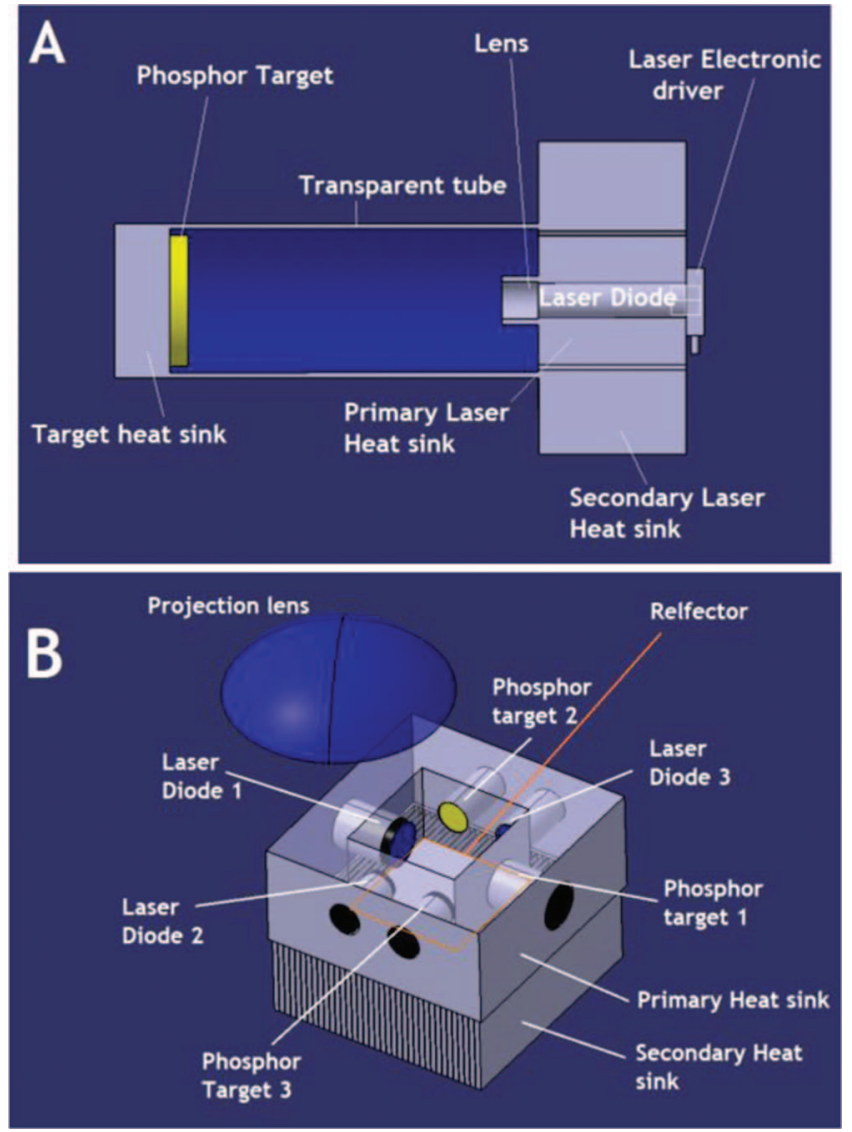

Figure 3. Structure of the Alpha (A) and Beta lamp (B).

Hence: $(3 \times \sim 365$ lumens) are lost due to the size of the lamp and the phosphor target housing.

d. Since we are only concerned about the performance and characterization of the phosphor target and not the lamp 'Beta', we can add the $(3 \times 365$ lumens $)$ lost due to the lamp structure back to the 1338 lumens which give $\sim 2400$ lumens.

Hence: Lamp 'Beta' is assumed to be generating 2400 lumens with three phosphor targets activated.

So, the $1338 \mathrm{~lm}$ was made equivalent to the value of $2400 \mathrm{~lm}$ (three lamp Alpha configuration read outs). It is also important to highlight the fact that light guide development and proper mirroring will need to be implemented in future designs of these lighting modules. However, the results from lamp 'Beta' are presented here for the objective of demonstrating potential future design concepts only. Application of laser activated light sources have been reported before, ${ }^{8-12}$ however our emphasis on this report revolves around the stability of emission properties and thermal management of the 'phosphor target' when high laser beam powers are concerned. Figure 13 demonstrates the lamps 'Alpha' and 'Beta' during operation. The data were obtained from the Lamp 'Beta' without the lens covering the lighting chamber structure within lamp. The thermal stability of the test lamps was monitored using a thermal camera and the emission spectrum and properties were monitored by an integrating sphere equipped with a spectrometer.

\section{Results}

Phosphor particle characterization.-The two phosphor materials (A \& B) were investigated using SEM along with EDS. As it can be seen from SEM results presented in Figure 4; type 'A' phosphor is composed of large particles ( 10 to 20 micrometres) while type ' $\mathrm{B}$ ' is composed of smaller agglomerated phosphor particles (agglomerates
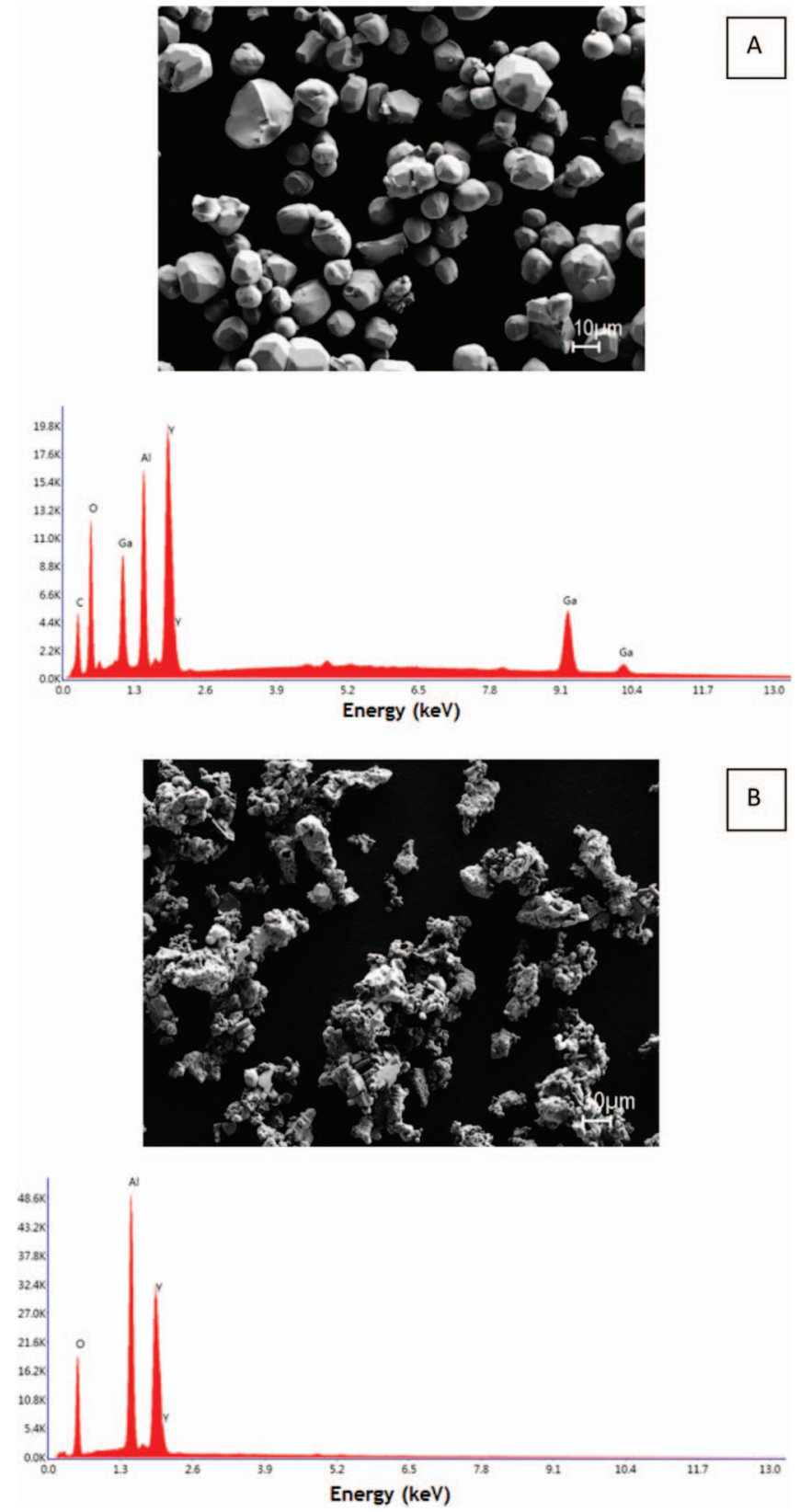

Figure 4. SEM \& EDS analysis of commercial phosphor types A (Top) and B (Bottom).

of 3 to 5 micrometres). EDS analysis indicates that gallium is present in phosphor A and is missing in phosphor B structure. The peak associated to carbon is due to the carbon substrate of the sample holder. The phosphors were also analyzed using X-ray powder diffraction (XRD) to evaluate their crystal structure; it was found that phosphor 'A' was industrial garnet phase with structure mostly matching PDF: 01-089-6659 with a formulation matching $\mathrm{Y}_{3} \mathrm{Ga}_{2} \mathrm{Al}_{3} \mathrm{O}_{12}$ and phosphor ' $\mathrm{B}$ ' demonstrated a structure matching PDF: 01-073-3190 with a formulation matching $\mathrm{Y}_{1.55} \mathrm{Yb}_{1.45} \mathrm{Al}_{5} \mathrm{O}_{12}$.

Up to $6 \mathrm{~W}$ beam power can be achieved by driving the diode at maximum current and voltage settings, we carried out most tests under $5 \mathrm{~W}$. The phosphor targets made from the two phosphors were then tested using a laser module with total power consumption of $\sim 22$ $\mathrm{W}$ generating a laser beam power of $\sim 5 \mathrm{~W}$ in an Alpha lamp configuration. Our laser diode assembly was designed so that we could focus and defocus the beam to various sizes, during these experiments we focused the beam so that at a distance of $12 \mathrm{~cm}$ away from 


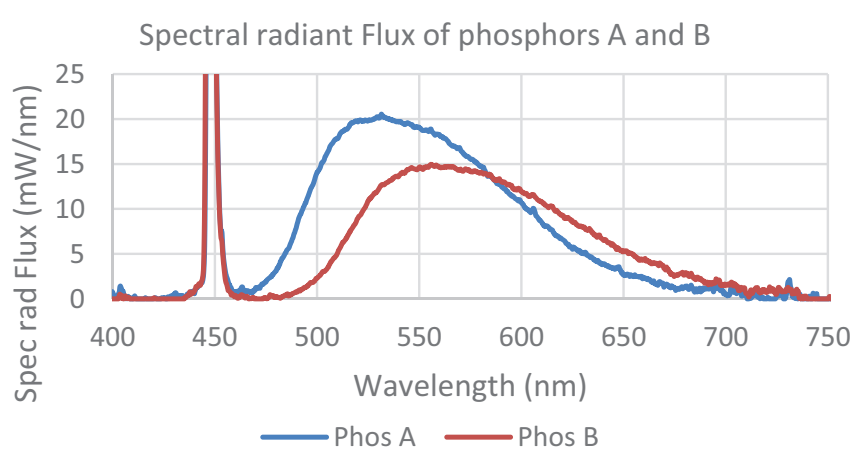

Chromaticity \& color temperature of Phosphor A and B

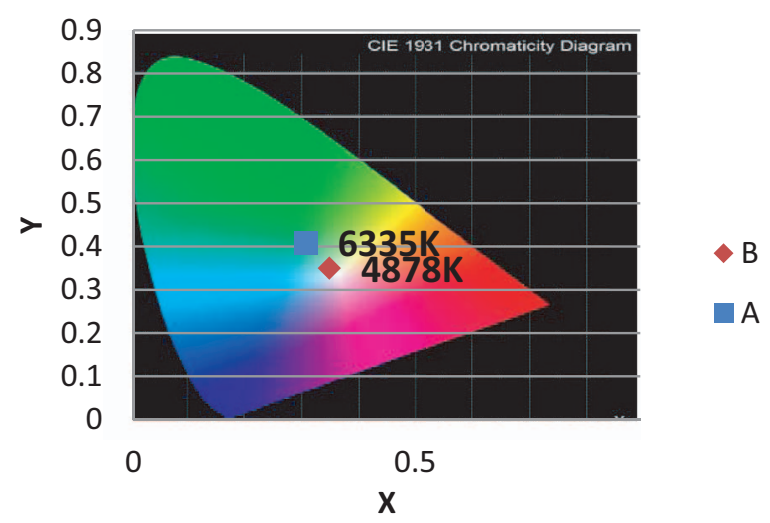

Figure 5. Comparison of the emission from the A and B phosphor types exposed to a $5 \mathrm{~W}$ laser beam power. Top: Spectral Radiant Flux, Bottom: Chromaticity and color temperature of the phosphors.

the phosphor target, the rectangular beam that was projected on to the target was $2 \times 5 \mathrm{~mm}$. The beam can be defocused or focused while maintaining the $5 \mathrm{~W}$ power output, hence the power density and the distance of the phosphor target from the source can be manipulated. Considering that currently the efficiency of the laser diodes is at $\sim 30 \%$ compared to the $>60 \%$ efficiency of LEDs, future advances in LD structures can lead to LDs which apart from being more efficient are not restricted to limited confinements when the remote phosphor target position is considered. The emission property of the two targets was obtained using an integrating sphere equipped with a spectrophotometer. The emission spectra of the two phosphors are presented in Figure 5.

The samples were tested by the lamp Alpha configuration system and were exposed to the beam for a period of 20 minutes. The luminous efficacy of the targets was measured at time 0 of being exposed to the beam and after 20 minutes, the results are presented in Figure 6. Although the phosphor target in lamp Alpha is thermally managed by having an independent heat sink, the heat sink is not connected to the main heat sink, hence over time it will elevate in temperature. Due to the rise of the target heat sink temperature we can see from Figure 6 that after 20 minutes an emission drop is observed. This drop in emission is only due to the temperature rise of the target and is not associated to phosphor degradation. Results from lamp Beta configuration will further elaborate on this issue. As we had reported previously, thermal management of the phosphor target when considering high laser beam powers is critical.

Phosphor concentration.-To demonstrate the relationship between the phosphor concentration within the inorganic matrix and the performance of the target, phosphor targets were made using only the type 'A' phosphor, without thermal management containing various percentages by weight of phosphor to matrix ratio. Table I presents

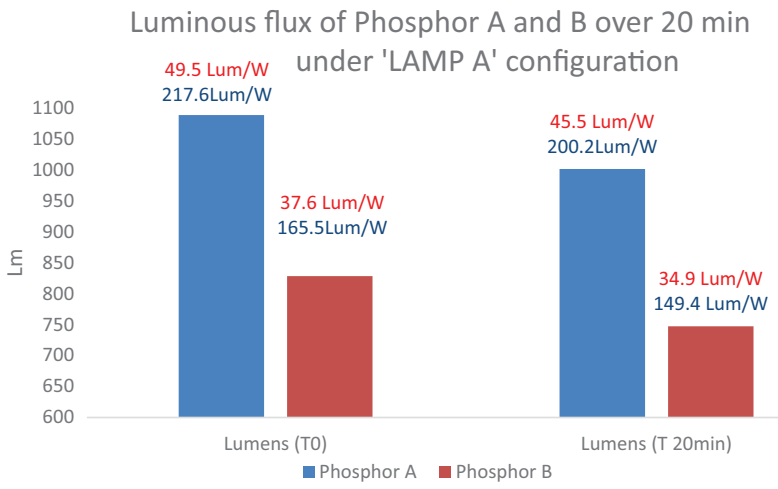

Figure 6. Phosphor A and B tested under lamp Alpha configuration. The luminous flux of the two phosphors at the start and after 20 min of exposure to the $5 \mathrm{~W}$ laser beam. The power consumption efficiency in lumens per watt in terms of wall plug efficiency is shown in red and per laser beam power in blue is presented on top of the relevant column.

the luminous flux and lumens per watt values of these samples when exposed to $6 \mathrm{~W}$ laser beam at time zero.

We had previously reported that up to $10 \%$ phosphor content in the target causes the luminous flux to increase with increasing phosphor concentration. ${ }^{7}$ Above $10 \%$ there doesn't seem to be a trend or relationship between the phosphor content and the luminous flux of the target. The reason for this from our understanding is related to the phosphor distribution and laser beam penetration into the matrix which is a constituent of the emissive target. The laser beam illuminated the target with normal beam incidence conditions ( 90 degrees). Our unique inorganic matrix material is a transparent material with phosphor particles dispersed within it; hence higher phosphor concentrations may be limiting the penetration of the laser beam in to the target but further studies specific to the refractive index of the target system need to be carried out to conclude a certain explanation for this observation. The distribution of the phosphor particles within the matrix was evaluated through surface mapping of the phosphor targets containing various phosphor contents. The maps were obtained by using laser Raman spectroscopy and using a single sharp band $\left(1300 \mathrm{~cm}^{-1}\right)$ in the Raman spectrum of the phosphor to study the distribution of the phosphor within the matrix, these tests were carried out to monitor the mixing technique and procedure of the phosphor and the matrix materials for even distribution of the phosphor particles.

The Raman spectrum of the phosphor A using a $633 \mathrm{~nm}$ laser is presented in Figure 7. As can be seen there are no peaks associated to the matrix while the phosphor has the Raman signature shown in Figure 7. Hence the phosphor distribution can be easily mapped

Table I. Phosphor (A) concentration within the matrix and luminous flux obtained when the samples are exposed to a $6 \mathrm{~W}$ laser beam (by operating the diode with a G2 lens at above threshold high currents. The luminous efficacy is calculated based on the $6 \mathrm{~W}$ beam powers from the $L D$.

\begin{tabular}{ccccc} 
& Luminous & Luminous & & \multicolumn{2}{c}{$\begin{array}{c}\text { Chromaticity } \\
\text { Index }\end{array}$} \\
\cline { 4 - 6 } concentration & flux Lm & efficacy Lm/W & $\mathrm{x}$ & $\mathrm{y}$ \\
\hline $10 \%$ & 1345 & 224.1 & 0.297 & 0.386 \\
$15 \%$ & 1157 & 192.8 & 0.290 & 0.363 \\
$20 \%$ & 1257 & 209.5 & 0.311 & 0.414 \\
$25 \%$ & 1288 & 214.6 & 0.310 & 0.406 \\
$30 \%$ & 1151 & 191.8 & 0.307 & 0.389 \\
$50 \%$ & 1116 & 186.0 & 0.321 & 0.429
\end{tabular}




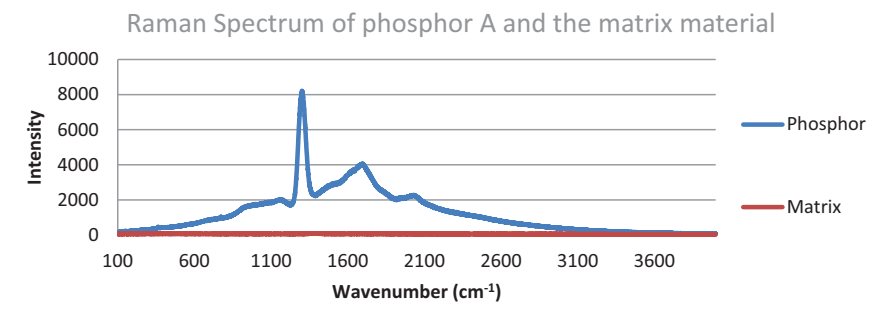

Figure 7. Raman spectrum of the phosphor A (blue) and the matrix (red).

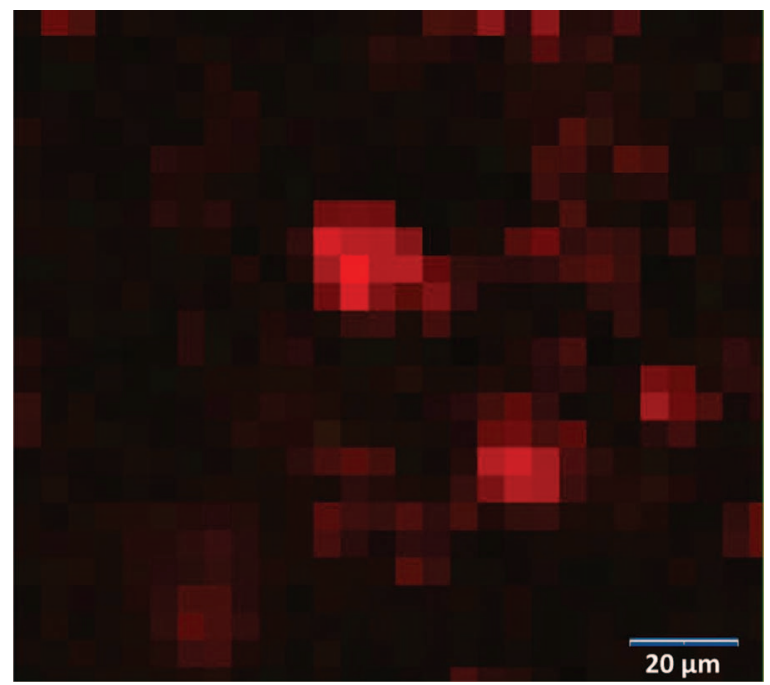

Figure 8. Surface mapping of the phosphor distribution over the target containing $10 \%$ phosphor. Mapping, Red intensity indicates phosphor presence and concentration.

(Figures 8 and 9) across the target surface using the very sharp peak at about $1300 \mathrm{~cm}^{-1}$.

From the results presented in Figures 8 and 9 and Table I, It can be seen that although the $35 \%$ sample has higher content of phosphor, its emission characteristics are not superior to the $10 \%$ sample. Here we have a dilemma; we need maximum number of phosphor particles exposed to the beam and we need deeper penetration of the beam into

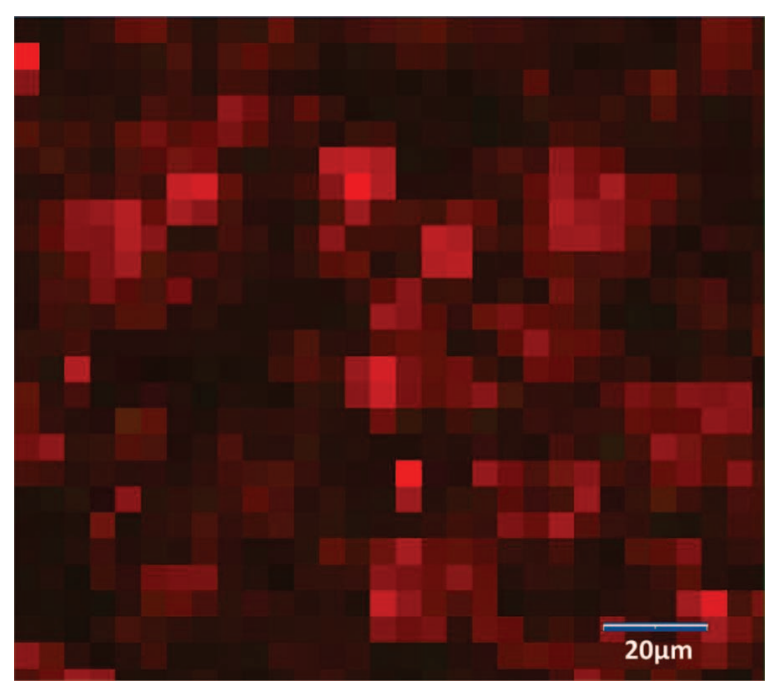

Figure 9. Phosphor distribution over the surface of the target containing 35\% phosphor. Red indicates phosphor presence. the Matrix to activate more phosphor particles dispersed within the matrix. We can assume that by increasing the concentration of the phosphors dispersed within the matrix we will have more phosphors being excited by the laser, however higher phosphor dispersion would also affect the penetration of the laser into the matrix by blocking its path. Hence there could be a trade-off between the amount of the phosphor that can be added to the matrix \& the depth of laser beam penetration into the matrix which can excite the phosphors dispersed deeper within the matrix.

This can possibly explain why increasing the phosphor concentration above $10 \%$ won't add any advantageous effects. These observations call for a separate thorough study on the diffraction properties of these targets in the future.

Phosphor combination.-The emission spectra of the SSL operated by LDs can easily be adjusted depending on the phosphor used. A combination of lasers and phosphors can be integrated to generate emission spectra tailored to specific demands. To demonstrate this possibility, a phosphor target was prepared so that the manipulation of the LD based SSL system's emission spectrum can be presented. Using commercial LED phosphors from the same commercial supplier, with peak emission bands of $530 \mathrm{~nm}$ and $650 \mathrm{~nm}$ a few targets were made and tested. The phosphors used were the phosphors A (530 $\mathrm{nm}$ ) which has already been discussed, along with a red metal silicon oxynitride phosphor with chemical formula $\mathrm{AE}_{\mathrm{z}}(\mathrm{Si}, \mathrm{O})_{\mathrm{x}} \mathrm{N}_{\mathrm{y}}: \mathrm{Eu}^{2+}$ according to the manufacturer, where $\mathrm{AE}$ is an Alkali earth element. These results are presented in Figure 10; combi 1 is the phosphor A, combi 2 is the red phosphor and combi 3 is a 50:50 (by mass) combination of both phosphors. Like LED remote phosphors, by altering the amount or ratio of the phosphors, the spectrum can be manipulated. To demonstrate this for LD excitation this test was carried out with the results shown in Figure 10.

Thermal monitoring of the phosphor target.-The performance of the phosphor targets activated by high power laser beams is extremely sensitive to the temperature of the target; the beam power can heat up the target and severely affect the output of the module. To elaborate on the importance of this concept; using the lamp Alpha configuration along with a phosphor target made from the phosphor 'A', the luminous efficacy of the target under various temperatures (as a result of the laser beam heating up the target) was monitored. The heat sink of the phosphor target was isolated from the main heat sink to allow gradual increase of the phosphor target's temperature. The temperature of the target along with power consumption and luminous flux of the test lamp were recorded. These results are presented in Table II and the plot of the relationship between the target

\section{Phosphor mix presentation}

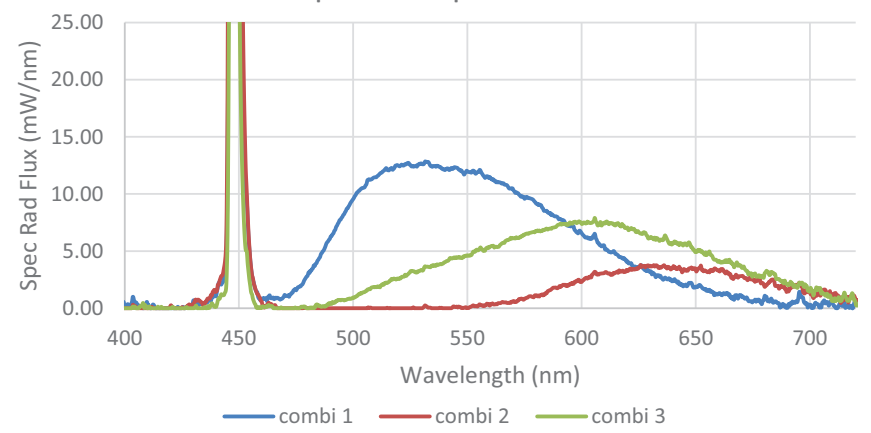

Figure 10. Emission characteristics of the SSL based on a LD which can easily be manipulated and tailored by application using various phosphor mixtures. The phosphor combinations were random and only made for presentation of this capability. Each combination contains random amount of three different phosphors. 


\begin{tabular}{|c|c|c|c|c|c|c|c|c|}
\hline $\begin{array}{l}\text { Time } \\
(\min )\end{array}$ & $\begin{array}{l}\text { Current } \\
\text { (A) }\end{array}$ & $\begin{array}{l}\text { Power } \\
\text { (W) }\end{array}$ & $\begin{array}{l}\text { Luminous } \\
\text { flux }(\mathrm{lm})\end{array}$ & $\begin{array}{l}\text { Luminous efficacy } \\
(\mathrm{Lm} / \mathrm{W}) \text { (wall plug) }\end{array}$ & Temp ${ }^{\circ} \mathrm{C}$ & $\begin{array}{l}\text { Luminous efficacy } \\
(\mathrm{Lm} / \mathrm{W}) \text { (beam) }\end{array}$ & \multicolumn{2}{|c|}{ Chromaticity index } \\
\hline 0 & 2.48 & 22.32 & 1093 & 48.9 & 20 & 218.6 & 0.307 & 0.413 \\
\hline 1 & 2.49 & 22.41 & 1086 & 48.4 & 21.7 & 217.2 & 0.306 & 0.408 \\
\hline 2 & 2.53 & 22.77 & 1077 & 47.2 & 23.2 & 215.4 & 0.305 & 0.408 \\
\hline 5 & 2.53 & 22.77 & 1063 & 46.6 & 26.8 & 212.6 & 0.305 & 0.406 \\
\hline 6 & 2.54 & 22.86 & 1060 & 46.3 & 28 & 212 & 0.306 & 0.406 \\
\hline 7 & 2.54 & 22.86 & 1052 & 46.0 & 31.5 & 210.4 & 0.305 & 0.405 \\
\hline 8 & 2.55 & 22.95 & 1036 & 45.1 & 32.5 & 207.2 & 0.305 & 0.407 \\
\hline 9 & 2.55 & 22.95 & 1035 & 45.0 & 33.7 & 207 & 0.305 & 0.406 \\
\hline 10 & 2.55 & 22.95 & 1034 & 45.0 & 34.5 & 206.8 & 0.306 & 0.407 \\
\hline
\end{tabular}

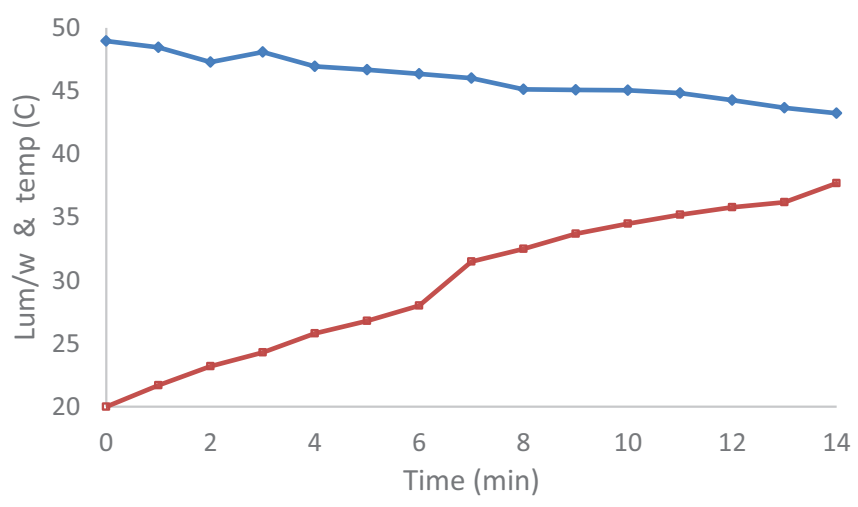

Figure 11. Thermal quenching of the device. [Blue: Lum/W (wall plug)], Red: Temperature ${ }^{\circ} \mathrm{C}$.

body temperature and luminous efficacy are presented in Figure 11. The temperature was monitored using a FLIR Thermacam PM695 thermal detection camera (Figure 12).

The thermal limitation associated to the Alpha structure can be avoided by means of connecting the phosphor heat sink into the main heat sink or an alternative thermal conducting body. It is a matter of module design on how the phosphor target can be kept cool. We decided to demonstrate such a design through the Beta configuration. Our approach in designing the Beta lamp was to incorporate three high power laser diodes and their associated phosphor targets into a single heat sink and a cooling fan. We specifically designed lamp Beta so that we can have a lamp which can maintain the low temperature required for the phosphor target so we can carry out an extended evaluation of the phosphor target performance without the thermal factor 'significantly' effecting the results. Specifically speaking the objective was to monitor possible degradation of the phosphor target when exposed to the laser beam for a long duration.

The advantage of the Beta lamp design is that the heat sinks of the phosphor and the laser modules are integrated and cooled effectively via a fan assisted heat sink. The test lamp Beta was operated for a period of six hours and the results are presented in Table III and Figure 14.

After the first six hour test, the lamp was then turned off and operated again after 24 hours for a period of 700 minutes to investigate possible damage to the phosphor targets. These results are presented in Figure 15. Due to the efficient thermal management applied in the design of lamp B, the emission from the lamp remains constant with only small fluctuations. Considering that this lamp was assembled using the inferior phosphor (B). It is apparent that by applying the type A phosphor or even more efficient phosphors, the performance of the lighting module can be enhanced even further.

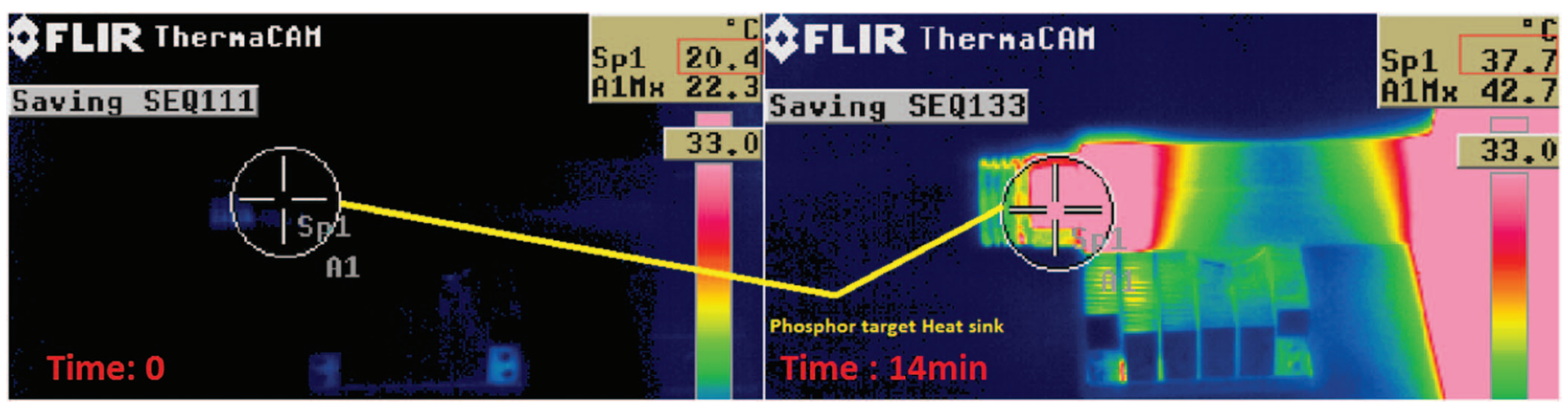

Figure 12. Temperature monitoring of the phosphor target's heat sink during operation was carried out with a thermal camera so that luminous efficacy of the lamp can be plotted and monitored alongside the temperature of the phosphor target against time, see Figure 11. Figure on the left illustrates the temperature of the target heat sink at time 0 which is 20.4 degrees Celsius and figure on the right demonstrates the target temperature after 14 minutes of lamp operation which is 37.7 degrees Celsius. 

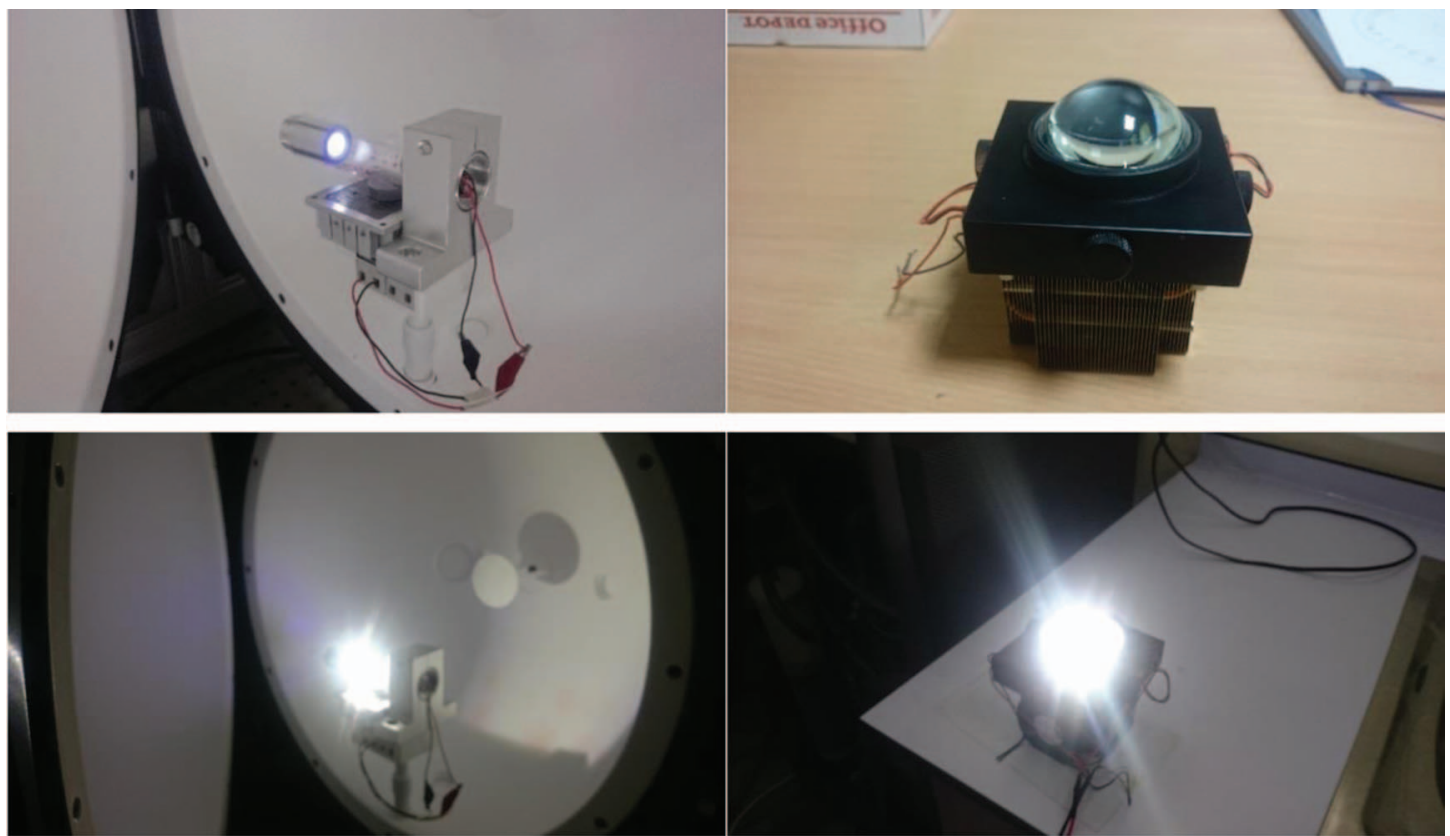

Figure 13. Left, Lamp Alpha and Right, Lamp Beta. Top: Off state, Bottom: On state

\begin{tabular}{|c|c|c|c|c|c|}
\hline $\begin{array}{l}\text { Time } \\
(\min )\end{array}$ & $\begin{array}{l}\text { Current } \\
\text { (A) }\end{array}$ & $\begin{array}{l}\text { Power } \\
\text { (W) }\end{array}$ & $\begin{array}{l}\text { Luminous } \\
\text { flux (lm) }\end{array}$ & $\begin{array}{l}\text { Luminous } \\
\text { efficacy } \\
(\mathrm{lm} / \mathrm{W}) \\
\text { (wall plug) }\end{array}$ & $\begin{array}{l}\text { Luminous } \\
\text { efficacy } \\
\text { (lm/W) } \\
\text { (beam) }\end{array}$ \\
\hline 0 & 7.35 & 66.15 & 2400.0 & 36.2 & 160 \\
\hline 30 & 7.3 & 65.7 & 2208.0 & 33.6 & 147.2 \\
\hline 60 & 7.32 & 65.88 & 2193.7 & 33.2 & 146.2 \\
\hline 90 & 7.32 & 65.88 & 2220.6 & 33.7 & 148.0 \\
\hline 120 & 7.32 & 65.88 & 2208.0 & 33.5 & 147.2 \\
\hline 180 & 7.32 & 65.88 & 2217.0 & 33.63 & 147.8 \\
\hline 210 & 7.33 & 65.97 & 2238.5 & 33.9 & 149.2 \\
\hline 270 & 7.32 & 65.88 & 2218.8 & 33.6 & 147.9 \\
\hline 300 & 7.32 & 65.88 & 2242.1 & 34.0 & 149.4 \\
\hline 330 & 7.31 & 65.79 & 2242.1 & 34.0 & 149.4 \\
\hline 390 & 7.32 & 65.88 & 2226.0 & 33.7 & 148.4 \\
\hline 420 & 7.31 & 65.79 & 2222.4 & 33.7 & 148.1 \\
\hline 450 & 7.31 & 65.79 & 2220.6 & 33.7 & 148.0 \\
\hline
\end{tabular}

Luminous efficacy of the Lamb B over six hours

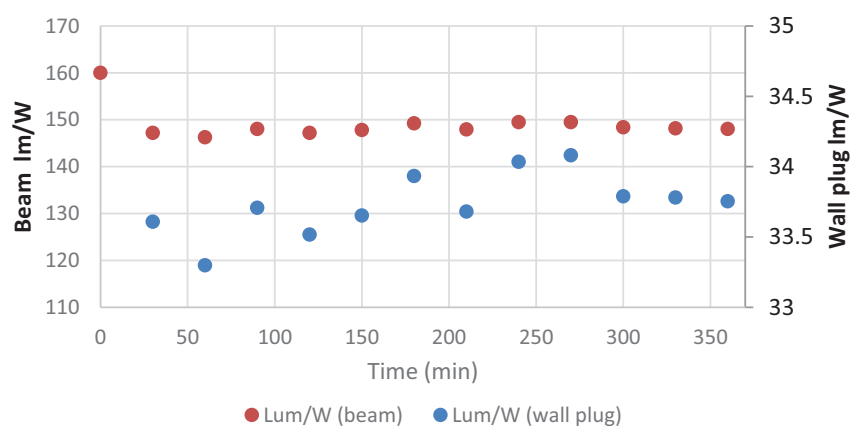

Figure 14. Stability of test lamp B performance in terms of wall plug efficiency and laser beam's power efficiency (15 W laser beam).

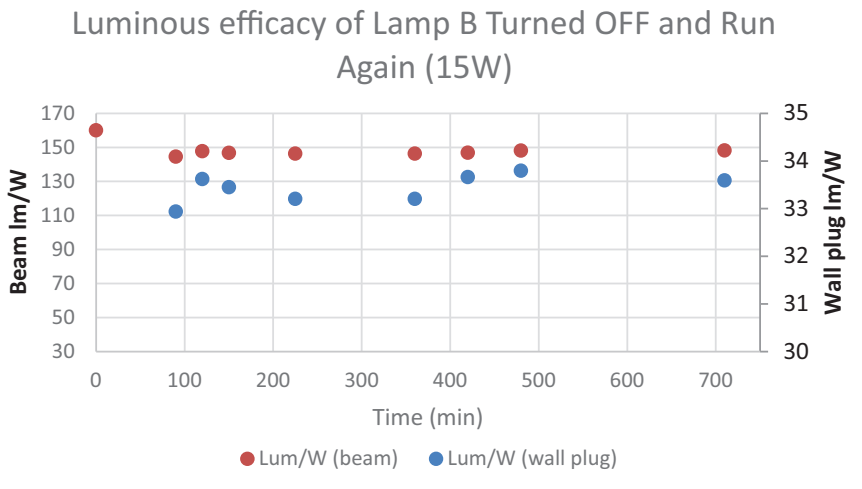

Figure 15. Luminous efficacy of the Lamp B; after the first 350 minutes run the lamp was cooled and then turned on and run for over 700 minutes.

\section{Conclusions}

The objective of this report was to demonstrate the potential that the future holds in the area of LD technology research and development. Laser diode based lighting is finding its way in to the commercial markets and here we have demonstrated that there is room for further research on using very high powered LDs $(5 \mathrm{~W})$ as the light engines of future SSL by designing phosphor targets capable of dissipating the thermal energy which is loaded into the phosphor by the laser beam. The efficiency of LD based SSLs will have room for improvement in two specific areas. These are: a. Phosphor formulation / phosphor screen structures used in these devices and b. developments in LD efficiency. We demonstrated that by varying the method of synthesis and composition of phosphor particle a significant increase in luminous efficacy value can be achieved; from $149 \mathrm{~lm} / \mathrm{W}$ to $200 \mathrm{~lm} / \mathrm{W}$. Further work on phosphor formulation and target structures along with structural design concepts associated to the lamp configuration can enhance these figures, which will benefit lighting systems based on both LEDs and LDs. ${ }^{8-12}$ The structure of the target and the blend of phosphor will enable tuneable high power emission characteristics. Since we have used a remote phosphor configuration when the LD 
eventually fails it can be easily replaced using our methodology thus enabling easy recycling or reapplication. However, as discussed earlier the major breakthrough will be reliant on production of future LDs with high efficiency which will significantly enhance the efficiency of SSL modules based on the LDs.

\section{Acknowledgments}

We thank Brunel University London for allocation of funds from EPSRC grant No. EP/K504208/1.

\section{References}

1. Y. C. Shen, G. O. Mueller, S. Watanabe, N. F. Gardner, A. Munkholm, and M. R. Krames, Appl. Phys. Lett., 91, 141101 (2007).

2. A. David and M. J. Grundmann, Appl. Phys. Lett., 97, 033501 (2010).
3. J. Iveland, L. Martinelli, J. Peretti, J. S. Speck, and C. Weisbuch, Phys. Rev. Lett., 110, 5 (2013).

4. J. J. Wierer, J. Y. Tsao, and D. S. Sizov, Laser. Photonics. Rev., 7(6), 963 (2013).

5. J. J. Wierer, J. Y. Tsao, and D. S. Sizov, Phys. Statu. Solidi. C, 11(3-4), 674 (2014).

6. Y. Ohno, Opt. Eng., 44, 111302 (2005).

7. A. Salimian, J. Silver, G. Fern, M. Evans, and R. Haghpanahan, ECS Journal of Solid State Science and Technology, 5(1), R3001 (2015).

8. A. Kristin, Michael Cantore, Shuji Nakamura, Steven P. DenBaars, and Ram Seshadri, AIP Advances, 3, 072107 (2013).

9. Alan Lenef, John Kelso, Maxim Tchoul, Oliver Mehl, Jörg Sorg, and Y. Zheng, "Thirteenth International Conference on Solid State Lighting," SPIE Proceedings, Vol. 9190 (18 Sep 2014).

10. U. Hartwig and M. Bruemmer, "Challenges for Reducing the Size of Laser Activated Remote Phosphor Light Engines for DLP Projection," in Classical Optics 2014, OSA Technical Digest (online) (Optical Society of America, 2014), paper ITh1A.6.

11. http://www.osram.com/osram_com/products/led-technology/specialty-lighting/ledmodules/itos/itos-phaser-3000/index.jsp?productId. Osram Corporation, visited 14.11.2015

12. Y. Tian, Journal of Solid State Lighting, 1, 11 (2014). 